\title{
Ten Simple Rules for Digital Government Transformation with Blockchain Smart Contracts
}

\author{
Chatphimuk Supinyo, Pongkorn Settasompop, Plubploy Jandaeng, and Surasak Phetmanee
}

\begin{abstract}
The digital transformation makes our government sustainable accomplishment. Smart contracts are the primary purpose of digital government transformation. In this paper, we present ten simple rules to enable users to be careful and use this technology correctly. The organisation can be achieved a successful transformation to the smart organisation. Our rules are presented in nonchronological order that you should do before you even think about blockchain with smart contracts. This paper also encourages a regular user to apply in the government that still have used a traditional transaction. The recommendation can follow the suggested solution in order to have a successful migration from the traditional organisation towards suitable blockchain smart contracts.
\end{abstract}

Index Terms-Blockchain, cryptocurrency, smart contracts.

\section{INTRODUCTION}

In this paper, we discuss how to use blockchain coupled with a contract called blockchain smart contracts. Smart contracts are the primary destination for digital government transformation. Many things that used to be analogue, then changed to various digital technologies have emerged so much to facilitate everyday life.

In order to understand the use of blockchain with smart contracts is increasing the growth of digital government transformation. In particular, the use and development of smart contracts are concern about algorithms and the law. As research from Treleaven et al. [1] addresses the issue of algorithms as artificial persons, with the necessary to formally verify and control algorithms. They discussed about blockchain technologies which support the currency and digital transactions for data manipulation.

Blockchain technologies invented for bitcoin and other cryptocurrencies, and they have potential in other areas. Blockchains are a method to order transaction in a distributed ledger. The core blockchain technologies are distributed ledger and smart contracts. The distributed ledger is a decentralised database where transactions are manipulation and secured by cryptographic sealing. The smart contracts are the rules that participants have collectively signed up to that confirm the evolution of the agreements in the distributed ledger. We can guarantee agreements when the pair of

Manuscript received April 30, 2020; revised July 12, 2020. This work was supported in part by Thammasat School of Engineering, Thammasat University.

Chatphimuk Supinyo, Pongkorn Settasompop, and Plubploy Jandaeng are with Thammasat University Programmes of Innovative Engineering, Thammasat University, Thailand (e-mail: chatphimuk.sup@dome.tu.ac.th, pongkorn.set@dome.tu.ac.th, and plubploy.jan@dome.tu.ac.th).

Surasak Phetmanee is with Electrical and Computer Engineering Department, Thammasat School of Engineering, Thammasat University, Thailand (e-mail: psurasak@engr.tu.ac.th). contract records are signed. Smart contract technology has the potential to automate the digital government transection. Although blockchain smart contracts are recently leading technology, most of the organisations still have used traditional contracts which cannot support the current change of trends such as new financial activities or support the growing future innovations. The key attributes are resilience, integrity, transparency, and unchangeable. To overcome these drawbacks, we offer a guide to best practice with the expectation that the reader is familiar with "economics" but has limited knowledge of blockchain technologies.

The digital transformation helps the government rise to success and sustainable. Digital technologies can transform the way we operate and deliver services to people. In this day, we talk about the strategies that will help us to change the traditional processes to our governments. What is the most effective way to use digital technologies to transform governments? We considered this question and found the blockchain smart contract is one of the keys to allow governments to break down the problems and policy issues. This paper encourages governments to start adopting this technology. Many governments in Thailand are planning to use the power of blockchain smart contracts.

We are a group of students who study in Software Engineering at the Programmes of Innovative Engineering, Faculty of Engineering, Thammasat University, Thailand. We found other experts or groups wrote many articles. The PLOS Ten Simple Rules collection inspires this paper. Philip E. Bourne wrote $48.6 \%$ of PLOS Ten Simple Rules articles. The ten simple rules methodology can represent the value and easy to understand. Firstly, we considered this topic about the rules for digital government transformation with blockchain smart contracts. We received multiple rules from our first discussion, but we ranked the rules to ten topics for writing this paper. Our results passed to the supervisor for evaluation and suggestion. Next, we read the literature and discussed with our supervisor about the detail of this article. Finally, we selected the writing style that could thoroughly explain in this domain. This paper wrote to focus on the audience who will read the rules and apply to their government. We present ten simple rules that we intend to enable users to be careful and use correctly. Our rules are presented in nonchronological order that you should do before you even think about blockchain with smart contracts.

We have organised the rest of this paper as follows. Section II describes ten simple rules for digital government transformation with blockchain smart contracts. Conclusion and future works are shown in Section III. The contributions are written on the last page of this paper. We hope this paper will help regular users to understand the blockchain smart contracts and success to transform their governments. 


\section{TEN SIMPLE RULES}

This paper presents the ten simple rules for digital government transformation with blockchain smart contracts. Table I shows our rules which you can follow before using blockchain smart contracts. For each rule, we present case studies, literature review, and further reading which are provided in the supplementary material.

TABLE I: THE TEN SIMPLE RULES

\begin{tabular}{ll}
\hline \hline No. & Rules Description \\
\hline 1. & Knowing smart contracts fundamental \\
2. & Choose a trustable wallet \\
3. & Do not forget your wallet ID and password \\
4. & Note your private key offline and keep in secret \\
5. & Choose appropriate smart contracts \\
6. & Choose trustable blockchain \\
7. & Be sure you have enough money before sign \\
8. & consistent information \\
9. & As a service provider, you must test a system thoroughly, \\
10. & If you use blockchain smart contracts correctly, then it \\
\hline \hline
\end{tabular}

\section{A. Rule 1: Knowing Smart Contracts Fundamental}

Before to use the blockchain smart contract, you must know about what is a blockchain smart contract and how to use a blockchain smart contract by a study from trustworthy information such as the internet articles based on facts not the opinion of the article owner. If you do not understand the work progress of a blockchain smart contract, you will be misunderstanding or errors of using a blockchain smart contract. It will affect yourself or the public and causing the person responsible for the impact that is caused by the user.

Studying blockchain smart contract before using is highly recommended because the blockchain smart contract is quite complicated. For example, we use a cryptocurrency wallet, and we do not know the meaning of the private key. It may not be able to use it safely.

In the article "smart contracts: the ultimate guide to blockchain smart contracts - learn how to use smart contracts for cryptocurrency exchange!" was written by Parker [2]. He said about how to use the smart contracts and how to use for cryptocurrency exchange. This article described the basic concept behind a smart contract that can use in situations. He guided readers to understand about what a smart contract, blockchain technologies, and cryptocurrency exchanges.

\section{B. Rule 2: Choose a Trustable Wallet}

The storage of monetary as cryptocurrency you must choose a wallet according to the experience of the user. We divided into two major types. First, the hot storage wallet is a software wallet which is suitable for those who do not want to hold a physical device that may keep in an online wallet. Second, the cold storage wallet keeps in a hardware wallet or writes in a paper. It is suitable for those who do not trust in keeping data in digital. Hardware wallet may come in the form of USB devices that are plugged into a computer to verify identity.

In wallet choosing, if you are choosing a not suitable wallet, it may cause problems. For example, the user always forgot username or password and let web browser keep your username and password. We recommend using a wallet online that can forget both username and password. It may prevent authorisation to access the account from users who tend to forget about the information in our heads. However, people who are good at storing things should choose a hardware wallet because it is more convenient for them.

In the article "method of making, securing, and using a cryptocurrency wallet" was written by Loera [3]. He said about the security of cryptocurrency. He presented a method of making, securing, and using a tangible cryptocurrency wallet. It was a printable medium that represented a public key and hidden a private key in invisible ink. The additional article "method and system for securing cryptocurrency wallet" was written by Rosca [4]. He also described the security of cryptocurrency wallet. He presented a method for securing cryptocurrency transactions on an online wallet. $\mathrm{He}$ recommended separating the transaction website from the hosting where executed cryptocurrency transactions.

\section{Rule 3: Do not Forget Your Wallet ID and Password}

Users use the online wallet, and they must not forget the ID and password of the wallet access. The only thing that gives us access to the online wallet is your ID and password. Therefore, we forget the ID and password like we forget the evidence. The rights to use to show ownership of that cryptocurrency wallet.

For example, news about the case of the facebook company was attacked by hackers. Therefore, on 27 September 2018, facebook had to reset the systems. The user who was reset must log in to the website or application again. However, many users forgot their username and password which makes them unable to login to the system. Several users cannot access authentication to the system since reset.

In the article "virtual wallet" was written by Boudreau [5]. He said about virtual features and benefits, which are also part of the article that highlights the importance of virtual wallet id and password recognition. The virtual wallet method provided a central portal for electronic commerce transactions that serviced end users. He implemented a central portal website that stored unique usernames and passwords for the virtual wallet.

\section{Rule 4. Note Your Private Key Offline and Keep in Secret}

A private key in the cryptocurrency wallet is comparable to what we hold to show ownership for the cryptocurrency wallet that we use. Therefore, if we know the private key of any cryptocurrency wallet, we can say that we are the owner of that cryptocurrency wallet. By keeping the private key, it should be more secure than the public key.

Keeping private key is essential and delicate because private must keep in secret. Many people may keep the private key in the service provider. If there is a reliable service provider, it may be safe. An online platform such as a cloud drive that has an authentication system to access the private key that you store like this. However, we do not forget the private key, so how we leave a safe in someone else's home. We could keep the private key at ourselves, which is not keeping in the digital platform such as writing in the physical paper and put in a safe box in a house or separate a paper and putting in a secret place. 
In the supplementary article "forward security in private key cryptography" was written by Bellare and Yee [6]. They said about private key cryptography which is also part of the article that highlights the private key. This paper provided a security concern about key sharing behaviour that cause the lack of security issues. They presented forward security techniques with the pseudorandom bit generator. This paper aimed to guarantee the strength of cryptographic algorithms for the private key cryptography.

\section{E. Rule 5: Choose Appropriate Smart Contracts}

This rule is essential because smart contracts are the primary purpose of digital government transformation. If we choose the appropriate smart contracts, we will integrate and use efficiently. Smart contracts have many types that include;

1) Code-is-contract is a natural language contract that can be encrypted.

\section{2) Hybrid contract is a first contract that combines a} computer code to make a contract or a code that changes to a paper contract.

3) Code as business logic is a popular contract and most common in everyday life. Most come in the form of daily spending because it has a consistent cycle.

For example, if we were to build a dormitory with monthly rental fees, we wanted to use blockchain smart contract technology as part of the system to collect monthly rent. We should carefully study whether the type of blockchain smart contract which is suitable for the type of contract we want to create. In this case, the form of contact will be a monthly financial transaction, so we should choose code as business logic to create the contract because of the same objectives.

In the article "smart contracts: the ultimate guide to blockchain smart contracts - learn how to use smart contracts for cryptocurrency exchange!" was written by Parker [2]. He said about how to use smart contracts and explain about a type of contract by explaining in suitable of contract.

\section{F. Rule 6: Choose Trustable Blockchain}

Nowadays, many companies are developing blockchain systems by choosing to use blockchain from reliable developers. It is a guarantee that will be no errors that affect the operation of the system. Alternatively, if an error occurs, developers are prepared to deal with to have less impact and can be modified for a short period.

Choosing a service provider or the developer of the blockchain system is another important thing to using blockchain smart contracts. Because developers who want to build a smart contract system, they need to implement a smart contract on blockchain technology. If we choose the blockchain incorrectly, it may cause the system that we created is not secure and suitable enough to use.

In the article "software reliability research" was written by Jelinski and Moranda [7]. They said about trust and selection of applications including observing for abnormalities in the software, selecting software from highly popular and self verified developers and company registration. They described the problems in various software anomalies and discussed about software reliability case studies. We can also apply software credibility principles to choose the blockchain smart contracts.

\section{G. Rule 7. Be Sure You have Enough Money before Sign}

In case a contract involves regular payment of expenses contract recipients. They should have to prepare enough money to pay in every cycle. Most of the contracts will specify the punishment. In case of paying or paying late contract fees, which will cause the rights that the contract recipient should receive may not be complete due to breach of contract in part.

For example, in the case that we receive a contract to pay electricity such as monthly water bills. We should always check how much and prepare enough money before the day of bank clearing. In doing so, both parties will be a mutual benefit. The contracting party will take benefit in terms of convenience, fast to pay for daily services by not waste time to pay at the bank. It also gets credit for making transactions from timely payments. Next, on the side of the contract, they will receive money on time. It means that you can manage your finances.

In the article "smart contracts: the ultimate guide to blockchain smart contracts - learn how to use smart contracts for cryptocurrency exchange! " was written by Parker [2]. He said about how to use smart contracts in type bill payment that has an advantage, including convenience and fast. However, you must have enough money every time. If the system has an error, it will take a long time to contact the staff and fixed it.

\section{H. Rule 8: Be Sure Your Contract Parties Understand Accurate and Consistent Information}

Every contracting party should have in a traditional or smart contract is that all parties must understand the details of the corresponding contract. Before making the contract, both parties should read and understand the content of the contract. They should find any queries or points that need to be resolved and notify other parties before agreeing to sign a contract. It relieves the doubts or makes corrections at that point to agree on both sides.

Making a blockchain smart contract is very important. It is not different from the understanding between the contract recipient and the contract provider. If not understanding the content of the agreed agreement, it may cause problems in the future. For example, the contract taker and the contract giver agree to deduct money from the account every mid-month. The giver means on the $15^{\text {th }}$ of every month, but the contract receiver thinks the mid-month is the day of that month divided by two. The contract recipient is not able to prepare the money to pay in time. It is resulting in a breach of contract.

In the article "evaluation of logic-based smart contracts for blockchain systems" was written by Idelberger et al. [8]. They said about the fundamental of smart contracts on the blockchain system that has a few rules or regulations. It should be used when using smart contracts between contract recipients and contract providers. They presented to use logic-based language for smart contracts where governance can ensure the system work and failure prevention. In the article provided the utility of logic-based smart contracts to bridge the gap between smart contracts and legal contracts. They proposed the use of logic-based smart contracts which combined with blockchain systems. 


\section{Rule 9: As a Service Provider, You Must Test a System thoroughly, and a Contract is Reliable for Everybody}

Blockchain smart contracts are one of the relatively new technologies. Therefore, a study of the use in various forms, including testing or trial a prototype. Checking errors will be generated after being used and editing before using the system. The experimentation of the prototype may specify when putting into the system. The main question is how the system affect? It may cause service providers to prepare measures to cope with the impact.

As a service provider, regardless of the type of product service providers should have a usage examination to see both the impact and the results. Service providers make the real product released to the market more efficient such as dividing the system version into beta and released for users to try. Because service provider testing may not cover the use of different forms in each user, this beta test will add to test cases from testers who are nearing the real users. That will become a real user when the service provider releases the real system.

In the article "a preliminary survey on software testing practices in Australia" was written by Ng et al. [9]. They said about an observation of a testing software before using, how it affects real users in Australia. The content also describes the effect on the future and summarised in the form of statistical data for easy reading. The details presented the survey on software testing practice from industries in their country which captured from 65 organisations between 2002 and 2003. They focused on five factors which include testing techniques, automated testing tools, metrics, standards, and software testing education. They found significant barriers in governments to adopt software reliability.

\section{J. Rule 10: If You Use Blockchain Smart Contracts Correctly Then It Should Be Continued}

The blockchain smart contracts are a way for users to save much time managing their daily tasks. As users, they do not have to manage the contract themselves with blockchain technology. The contract will be safer and more confidential, which will result in users have enough confidence in making contracts to dare to enter into contracts related to financial transactions. Therefore, if we can use blockchain smart contracts effectively then it should be continued.

Using the blockchain smart contracts in the beginning, it can be difficult because we have to spend a lot of effort and time to study information and regulations since rule one until rule nine. However, we can use blockchain smart contracts and get excellent benefits, and we should choose to continue to use. It will help to save time in making or managing various contracts in daily life.

In the previous article "smart contracts: the ultimate guide to blockchain smart contracts - learn how to use smart contracts for cryptocurrency exchange! " was written by Parker [2]. In this article has a guide to get a user to know about smart contracts and use knowledge of smart contract for efficient and secure in the long term. However, we strongly recommend updating the technology of blockchain smart contracts closely as it is still a new technology that is developing rapidly. Our primary concept is delivered the simple rules for users who want to transform governments with blockchain smart contracts.

\section{CONCLUSION}

The digital government transformation is the only way to improve our organisation successful and sustainable in the next generation. The digital transformation boosts the government for success and sustainable. Digital technologies can transform the way we operate and deliver services to people. The government will reduce unnecessary cost, increase more productivity, build up the secure transaction, and get more customer satisfaction. Blockchain smart contracts are one of the disruptive technologies that have several benefits such as secure transaction, low cost, private authentication and scalable. Blockchain technologies address distributed ledger and smart contracts. The distributed ledger is a distributed database where transactions are manipulation such as create, read, update, and delete. Furthermore, transactions are secured by cryptographic sealing. The smart contracts are laws that participants have signed up to confirm the evolution of agreements in the distributed ledger. These simple rules should be introduced into the transforming government in order to use for best practices and overcome several drawbacks from the traditional problems.

We set out to write this paper by using the methodology from PLOS Ten Simple Rules collection. We intend to enable users understanding the use of blockchain smart contracts and bring it to transform their governments. Our rules are presented in nonchronological order that recommends thinking before using blockchain with smart contracts. The results support the hypothesis that our rules can be used to follow when you use blockchain smart contracts. However, this is the early adoption era of digital transformation, and we recommend preparing for new updates. In conclusion, this paper also encourages a regular user to apply in the government that still have used a traditional transaction. The recommendation can follow the suggested solution in order to have a successful migration from the traditional organisation towards suitable blockchain smart contracts.

For future works, we concern more extensive analysis mechanisms and develop new proposals to investigate different methods. The ideas have to consider and brainstorm in the next class year. We are designed to compare the performance with this paper. We will extend our findings from this study into there topics. First, we have prepared a survey on how to guarantee and validation the correct of blockchain smart contracts. In a real situation, we have to consider many factors that can affect our system. This activity will ensure that proposed rules can be used well in the governments. Second, we have implemented this technology in our organisation, and we will observe the benefits of this technology. This work targets to examine the behaviour when the transformed governments bring services to people. They are satisfaction or not satisfaction with the blockchain smart contracts. Third, we will expand the use of blockchain smart contacts to other fields such as education, finance, and software security. When we discussed the rules for using smart contracts, we found this technology can be applied to many fields. So, we are interested in applying the blockchain technology in other areas. However, this paper is not an ultimate guide for using blockchain smart contracts, we hope this work will provide simple rules to help people who aim to use this technology correctly and successfully for the digital government transformation. 


\section{CONFLICT OF INTEREST}

The authors declare no conflict of interest.

\section{AUTHOR CONTRIBUTIONS}

Chatphimuk Supinyo, Pongkorn Settasompop, and Plubploy Jandaeng conducted this research under the supervision. We reviewed the literature and wrote simple ten rules on the first draft paper. We prepared the presentation to talk at the conference. Surasak Phetmanee supervised and guided us about the research methodologies. He wrote the missing details for all sections and helped to edit the final draft paper. All authors had approved the final version.

\section{ACKNOWLEDGMENT}

We thank editors who cleaned up and corrected any errors that we missed on this manuscript. We thank reviewers or mentors who read and suggested beneficial ideas for this work. We appreciate the IJCTE journal publishers who published this paper. We thank our supervisor and friends who taught and discussed the approaches to this project. We acknowledge all researchers who did previous research works that we used the important information in the part of our work. Finally, we thank the members of our family who lent our support while we did the project or wrote the manuscript.

\section{REFERENCES}

[1] P. Treleaven, J. Barnett, and A. Koshiyama, "Algorithms: Law and regulation," Computer, vol. 52, issue 2, pp. 32-40, February 2019.

[2] T. Parker, Smart Contracts: The Ultimate Guide to Blockchain Smart Contracts - Learn How to Use Smart Contracts for Cryptocurrency Exchange! Create Space Independent Publishing Platform, 2016.

[3] A. Loera, "Method of making, securing, and using a cryptocurrency wallet," U.S. Patent Application No. 14/178,234, August 13, 2015.

[4] A. Rosca. "Method and system for securing cryptocurrency wallet," U.S. Patent Application No. 14/848,294, March 10, 2016.

[5] B. Boudreau, "Virtual wallet," U.S. Patent Application No. 13/736,014, July 11, 2013.

[6] M. Bellare and Y. Bennet, "Forward-security in private-key cryptography," in Proc. Cryptographers' Track at the RSA Conference, Springer, Berlin, Heidelberg, 2003, pp. 1-18.

[7] Z. Jelinski and P. Moranda, "Software reliability research," in Statistical Computer Performance Evaluation, Academic Press, 1972, pp. $465-484$.

[8] F. Idelberger, G. Governatori, R. Riveret, and G. Sartor, "Evaluation of logic-based smart contracts for blockchain systems," in Proc.

International Symposium on Rules and Rule Markup Languages for the Semantic Web, Springer, Cham, 2016, pp. 167-183.

[9] S. P. Ng, T. Murnane, K. Reed, D. Grant, and T. Y. Chen, "A preliminary survey on software testing practices in Australia," in Proc. Australian Software Engineering Conference, 2004, pp. 116-125.

Copyright $(\odot 2020$ by the authors. This is an open access article distributed under the Creative Commons Attribution License which permits unrestricted use, distribution, and reproduction in any medium, provided the original work is properly cited (CC BY 4.0).
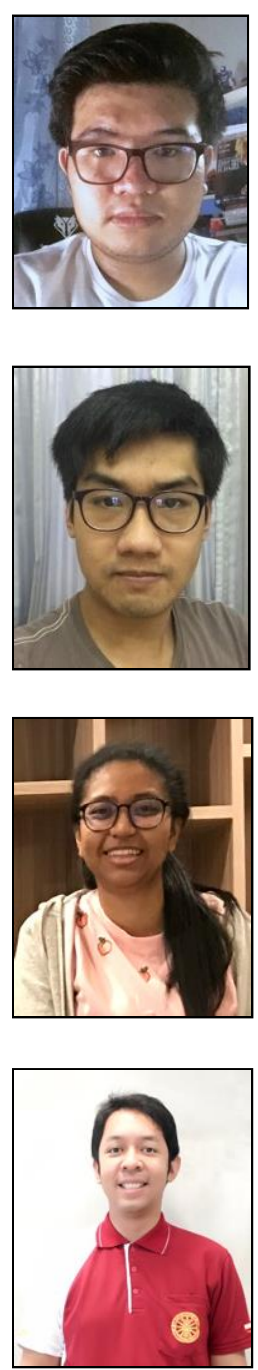

Chatphimuk Supinyo is a fourth-year student in software engineering at the Faculty of Engineering, Thammasat University. $\mathrm{He}$ has experience in software developer at many companies as an intern student. The main focus of his research has been on the theory of simple rules for digital government transformation with blockchain smart contracts which make the government sustainable accomplishment. He is a key contributor who has invented this paper.

Pongkorn Settasompop is a fourth-year student in software engineer at the Faculty of Engineering, Thammasat University. He is interested in the areas of software development, machine learning, and artificial intelligence. He is a key contributor for this research about blockchain, which includes fundamental of blockchain, how to use blockchain and how can we trust a blockchain with ten simple rules.

Plubploy Jandaeng is a fourth-year student in software engineering at the Faculty of Engineering, Thammasat University. She is interested in the areas of software engineering, software testing, and software security. She conducted this research with the research team and supervisor. She is a key contributor who has invented this paper.

Surasak Phetmanee is a lecturer in software engineering and the director of Computer and Information Center at the Faculty of Engineering, Thammasat University. He is a project supervisor who controlled the research methodology. His research focused on the topic of algorithmic trading, machine learning, and software engineering. He has been long interested in the development of financial software tools to support both academic and industry. 